\title{
Erratum
}

\section{On the heuristic of approximating polynomials over finite fields by random mappings}

\author{
[Int. J. Number Theory, Vol. 12, No. 7 (2016) 1987-2016] \\ Rodrigo S. V. Martins \\ Departamento Acadêmico de Matemática \\ UTFPR, Rua Marcilio Dias 635 \\ 86812-460, Apucarana, PR, Brazil \\ rodrigomartins@utfpr.edu.br \\ Daniel Panario \\ School of Mathematics and Statistics \\ Carleton University, Canada \\ 1125 Colonel By Drive \\ Ottawa, ON, Canada K1S 5B6 \\ daniel@math.carleton.ca
}

Published 15 July 2016

The concept of general polynomials was introduced in [1], where Birch and Swinnerton-Dyer proved an asymptotic estimate on the value set of these polynomials; see Definition 2.18 and Theorem 2.19. In [3] the author proves an enumeration result regarding the class of polynomials satisfying the conclusion of Theorem 2.19. More precisely, it is proved in [3] that the probability of a random uniform polynomial of degree $d$ over $G F(q)$ satisfies the condition in Theorem 2.19 is asymptotically 1 , as $q$ approaches infinity. As far as we know this class may include polynomials that are not general, since it is not known if the reciprocate of Theorem 2.19 holds.

In this erratum, we clarify that the concept of general polynomials of $[1,3]$ cannot, as far as we know, be used interchangeably, as was the case in the published version of this paper. The points below represent the significant changes in the content of the paper. We refer to general polynomials in this document strictly in the sense of $[1]$.

- Theorem 2.20 still holds for general polynomials, but it is proved using the results of $[1,2]$.

- Both proofs of item (iv) of Theorem 2.21 still hold, but the estimate for the value set of the polynomials in question is provided by the combination of Theorem 2.20 and the results of [3]. 
- As far as we know Theorem 2.26 is not proved for general polynomials. Theorems 2.27 and 2.28 hold under the assumption that the number $N$ of general polynomials of the form $f(x)=a_{d} x^{d}+a_{d-1} x^{d-1}+\cdots+a_{1} x+a_{0}$ satisfies $N=q^{d+1}(1+O(1 / q))$, as $q$ approaches infinity.

It is worth noting that the assumption above is motivated by the fact that Birch and Swinnerton-Dyer mention in [1] that they obtain simple sufficient conditions for a polynomial to be general and, "though not necessary, these are in fact satisfied by almost all polynomials."

\section{References}

[1] B. J. Birch and H. P. F. Swinnerton-Dyer, Note on a problem of Chowla, Acta Arith. 5(4) (1959) 417-423.

[2] S. Cohen, The distribution of polynomials over finite fields, Acta Arith. 17(3) (1970) 255-271.

[3] K. S. Williams, On general polynomials, Canad. Math. Bull. 10(4) (1967) 579-583. 